\title{
THE DIOPHANTINE PROBLEM FOR POLYNOMIAL RINGS AND FIELDS OF RATIONAL FUNCTIONS ${ }^{1}$
}

\author{
BY \\ J. DENEF $^{2}$
}

\begin{abstract}
We prove that the diophantine problem for a ring of polynomials over an integral domain of characteristic zero or for a field of rational functions over a formally real field is unsolvable.
\end{abstract}

1. Introduction. ${ }^{3}$ During the last thirty years much work has been done to prove that the elementary theory of various rings is undecidable; see J. Ax [1], Yu. Eršov [9], [10], A. Malcev [14], Yu. Penzin [15], J. Robinson [17]-[20], R. M. Robinson [21], [22] and A. Tarski [23].

After M. Davis, Yu. Matijasevič, H. Putnam and J. Robinson (see, e.g., [4], [6]) proved that the existential theory of $\mathbf{Z}$ is undecidable, it is natural to ask whether the existential theory of various other rings is undecidable too.

Let $R$ be a commutative ring with unity and let $R^{\prime}$ be a subring of $R$. We say that the diophantine problem for $R$ with coefficients in $R^{\prime}$ is unsolvable (solvable) if there exists no (an) algorithm to decide whether or not a polynomial equation (in several variables) with coefficients in $R^{\prime}$ has a solution in $R$.

In [7] we proved that the diophantine problem for the ring of algebraic integers in any quadratic extension of $\mathbf{Q}$ is unsolvable, and recently we have extended this to some more algebraic integer rings. For some very interesting related results, see L. Lipshitz [13].

The main results of this paper are:

THEOREM A. Let $R$ be an integral domain of characteristic zero; then the diophantine problem for $R[T]$ with coefficients in $\mathrm{Z}[T]$ is unsolvable. $(R[T]$ denotes the ring of polynomials over $R$, in one variable $T$.)

THEOREM B. Let $K$ be a formally real field, i.e. -1 is not the sum of squares

Received by the editors June $20,19 / \prime$

AMS (MOS) subject classifications (1970). Primary 02G05, 10N05, $10 \mathrm{~B} 99$.

Key words and phrases. Hilbert's tenth problem, unsolvable problems, diophantine equations.

${ }^{1}$ Dedicated to Professor L. P. Bouckaert on the occasion of his seventieth birthday.

${ }^{2}$ This work has been supported by the "Nationaal Fonds voor Wetenschappelijk Onderzoek". It was done at Harvard University, whose generous hospitality I greatly appreciate. I am also grateful to M. Boffa and R. M. Robinson for simplifying the proof of Lemma 2.1.

${ }^{3}$ We use the following notations: $\mathbf{N}$ is the set of natural numbers; $\mathbf{Z}$ is the ring of integers; $\mathbf{Q}$ is the field of rationals; $\mathbf{R}$ is the field of real numbers; and $\mathbf{C}$ is the field of complex numbers. 
in $K$. Then the diophantine problem for $K(T)$ with coefficients in $\mathbf{Z}[T]$ is unsolvable. $(K(T)$ denotes the field of rational functions over $K$, in one variable T.)

We prove Theorem $\mathrm{A}$ in $\S 2$ and Theorem $\mathrm{B}$ in $\S 3$.

It is obvious that the diophantine problem for $R[T]$ with coefficients in $\mathbf{Z}$ is solvable if and only if the diophantine problem for $R$ with coefficients in $\mathbf{Z}$ is solvable. And the same holds for $K(T)$. (An algebraic closed field, a real closed field, the ring of $p$-adic integers and the ring of formal power series over a decidable field of characteristic zero are examples of rings whose diophantine problem with coefficients in $\mathbf{Z}$ is solvable.)

R. M. Robinson [21] proved for any integral domain $R$ that the elementary theory of $R[T]$ is undecidable. M. Davis and H. Putnam [5] proved that the diophantine problem for $\mathbf{Z}[T]$ with coefficients in $\mathbf{Z}[T]$ is unsolvable. But, after that the diophantine problem for $\mathbf{Z}$ was proved unsolvable, it becomes trivial that the diophantine problem for $\mathbf{Z}[T]$ with coefficients in $\mathbf{Z}$ is unsolvable.

A. Malcev [14] and A. Tarski [23] proved that the elementary theory of $K(T)$ is undecidable when $K$ is a real closed field. A simpler proof of this result has been given by J. Robinson [20]. Later R. M. Robinson [22] extended this result to any formally real field $K$. Yu. Eršov [9] and Yu. Penzin [15] proved the undecidability of the elementary theory of $K(T)$ when $K$ is a finite field.

If $K$ is a formally real field then so is $K(T)$; thus Theorem B is also true for fields of rational functions in several variables.

It is interesting to compare our work with a result of J. Becker and L. Lipshitz [2]: The diophantine problem for $\mathrm{C}\left[\left[T_{1}, T_{2}\right]\right]$ (i.e. the ring of formal power series over $\mathbf{C}$, in the variables $T_{1}$ and $T_{2}$ ) with coefficients in $\mathbf{Z}\left[T_{1}, T_{2}\right]$ is solvable, although the elementary theory of $\mathrm{C}\left[\left[T_{1}, T_{2}\right]\right]$ is undecidable (see Eršov [10]).

Let $R$ be a commutative ring with unity and let $D\left(x_{1}, \ldots, x_{n}\right)$ be a realtion in $R$. We say that $D\left(x_{1}, \ldots, x_{n}\right)$ is diophantine over $R$ if there exists a polynomial $P\left(x_{1}, \ldots, x_{n}, y_{1}, \ldots, y_{m}\right)$ over $R$ such that for all $x_{1}, \ldots, x_{n}$ in $R$ :

$$
D\left(x_{1}, \ldots, x_{n}\right) \leftrightarrow \exists y_{1}, \ldots, y_{m} \in R: P\left(x_{1}, \ldots, x_{n}, y_{1}, \ldots, y_{m}\right)=0 .
$$

We have the same definition for subsets of $R$ by regarding them as 1-ary relations. Let $R^{\prime}$ be a subring of $R$ and suppose $P$ can be chosen such that its coefficients lay in $R^{\prime}$, then we say that $D\left(x_{1}, \ldots, x_{n}\right)$ is diophantine over $R$ with coefficients in $R^{\prime}$.

If $R$ is an integral domain and if $D_{1}$ and $D_{2}$ are diophantine over $R[T]$ with coefficients in $\mathrm{Z}[T]$, then also $D_{1} \vee D_{2}$ and $D_{1} \wedge D_{2}$ are diophantine 
over $R[T]$ with coefficients in $\mathbf{Z}[T]$; indeed,

$$
P_{1}=0 \vee P_{2}=0 \leftrightarrow P_{1} P_{2}=0 \quad \text { and } P_{1}=0 \wedge P_{2}=0 \leftrightarrow P_{1}^{2}+T P_{2}^{2}=0 .
$$

Moreover, the same holds for $K(T)$.

In this paper we prove also:

Proposition 1. Let $R$ be an integral domain of characteristic zero. Suppose there exists a subset $S$ of $R$ which contains $\mathbf{Z}$ and which is diophantine over $R[T]$; then $\mathbf{Z}$ is diophantine over $R[T]$. In particular, this is true when $R$ contains $\mathbf{Q}$.

Proposition 2. Let $K$ be a formally real field. Suppose there exists a subset $S$ of $K$ which contains $\mathbf{Z}$ and which is diophantine over $K(T)$; then $\mathbf{Z}$ is diophantine over $K(T)$. In particular, this is true when $K$ contains the real closure of $\mathbf{Q}$.

In [8] we have proved that a relation is diophantine over $\mathbf{Z}[T]$ if and only if it is recursively enumerable. M. Boffa noticed that for a nondenumerable language the situation can be very different:

Corollary (M. Boffa). Every subset $D$ of $\mathbf{N}$ is diophantine over $\mathbf{R}[T]$.

PRoof. Let $r$ be the real number $r=\sum_{n=0}^{\infty} a_{n} / 10^{n+1}$, where $a_{n}=0$ for $n \in D$ and $a_{n}=1$ for $n \notin D$. Then we have

$$
n \in D \leftrightarrow n \in \mathbf{N} \wedge \exists p, m \in \mathbf{N}:\left(m=10^{n} \wedge 0 \leqslant m r-p<\frac{1}{10}\right) .
$$

But $\mathbf{Z}$ is diophantine over $\mathbf{R}[T]$ by Proposition 1 , and every recursively enumerable relation in $\mathbf{Z}$ is diophantine over $\mathbf{Z}$ (see, e.g., [4], [6]). Thus, using elementary algebra, we see that $D$ is diophantine over R[T]. Q.E.D.

2. Polynomial rings. Let $R$ be any integral domain of characteristic zero. We consider the Pell equation

$$
X^{2}-\left(T^{2}-1\right) Y^{2}=1
$$

over $R[T]$. Let $U$ be an element in the algebraic closure of $R[T]$ satisfying

$$
U^{2}=T^{2}-1
$$

Define two sequences $X_{n}, Y_{n}, n=0,1,2, \ldots$, of polynomials in $\mathbf{Z}[T]$, by setting

$$
X_{n}+U Y_{n}=(T+U)^{n} \text {. }
$$

We prove that Lemma 2.2 of M. Davis and H. Putnam [5] remains true when $\mathbf{Z}$ is replaced by $R$ :

LEMMA 2.1. The solutions of (1) in $R[T]$ are given precisely by

$$
X= \pm X_{n}, \quad Y= \pm Y_{n}, \quad n=0,1,2, \ldots
$$


Proof. (1) is equivalent to

$$
(X-U Y)\left(X+U^{V}\right)-1
$$

From (3) and (2) follows

$$
X_{n}-U Y_{n}=(T-U)^{n}=(T+U)^{-n}
$$

Hence the $X_{n}, Y_{n}$ are solutions of (1).

Conversely, suppose $X$ and $Y$ in $R[T]$ satisfy (1). Let us parametrise the curve (2) by

$$
T=\frac{t^{2}+1}{t^{2}-1}, \quad U=\frac{2 t}{t^{2}-1} .
$$

The rational functions $X+U Y$ and $X-U Y$ in $t$ have poles only at $t= \pm 1$. Moreover (4) implies they have zeroes only at $t= \pm 1$. Hence

$$
X+U Y=c\left(\frac{t+1}{t-1}\right)^{m}=c(T+U)^{m}, \quad c \in R, m \in \mathbf{Z} .
$$

Thus also $X-U Y=c(T-U)^{m}$. But substituting this in (4) gives $c^{2}=1$, which proves the lemma by (3). Q.E.D.

Throughout this section we write $V \sim W$ to denote that the polynomials $V$ and $W$ in $R[T]$ take the same value at $T=1$. Notice that the relation $Z \sim 0$ is diophantine over $R[T]$ with coefficients in $Z[T]$, indeed

$$
Z \sim 0 \leftrightarrow \exists X \in R[T]: Z=(T-1) X .
$$

The following lemma was used by M. Davis and H. Putnam [5, Lemma 2.3] too:

LEMMA 2.2. We have $Y_{n} \sim n$, for $n=0,1,2, \ldots$.

Proof. From (3) and (2) follows

$$
Y_{n}=\sum_{\substack{i=1 \\
i \text { odd }}}^{n}\left(\begin{array}{c}
n \\
i
\end{array}\right)\left(T^{2}-1\right)^{(i-1) / 2} T^{n-i}
$$

Substitute now $T=1$. Q.E.D.

Let us define the 1-ary relation $\operatorname{Imt}(Y)$ in $R[T]$ by

$$
\operatorname{Imt}(Y) \leftrightarrow Y \in R[T] \wedge \exists X \in R[T]: X^{2}-\left(T^{2}-1\right) Y^{2}=1 .
$$

LEMMA 2.3. We have:

(i) The relation $\operatorname{Imt}(Y)$ is diophantine over $R[T]$ with coefficients in $Z[T]$.

(ii) If $Y$ satisfies $\operatorname{Imt}(Y)$, then there exists an integer $m$ such that $Y \sim m$.

(iii) For every integer $m$ there exists a polynomial $Y$ satisfying $\operatorname{Imt}(Y)$ and $Y \sim m$.

Proof. This follows at once from Lemmas 2.1 and 2.2. 
Proof of Theorem A. There exists an algorithm to find for any polynomial $P\left(z_{1}, \ldots, z_{n}\right)$ over $\mathbf{Z}$, a polynomial $P^{*}\left(Z_{1}, \ldots, Z_{m}\right)$ over $\mathbf{Z}[T]$ such that

$$
\begin{aligned}
\exists z_{1}, \ldots, z_{n} \in \mathbf{Z}: P\left(z_{1}, \ldots, z_{n}\right)=0 \\
\leftrightarrow \exists Z_{1}, \ldots, Z_{m} \in R[T]: P^{*}\left(Z_{1}, \ldots, Z_{m}\right)=0 .
\end{aligned}
$$

Indeed by Lemma 2.3 we have

$$
\begin{aligned}
& \exists z_{1}, \ldots, z_{n} \in \mathbf{Z}: P\left(z_{1}, \ldots, z_{n}\right)=0 \leftrightarrow \exists Z_{1}, \ldots, Z_{n} \in R[T]: \\
&\left(\operatorname{Imt}\left(Z_{1}\right) \wedge\right.\left.\cdots \wedge \operatorname{Imt}\left(Z_{n}\right) \wedge P\left(Z_{1}, \ldots, Z_{n}\right) \sim 0\right) .
\end{aligned}
$$

Since Imt and $\sim$ are diophantine over $R[T]$ with coefficients in $Z[T]$, we easily obtain a polynomial $P^{*}$ satisfying (5). Hence if the diophantine problem for $R[T]$ with coefficients in $\mathrm{Z}[T]$ would be solvable, then the diophantine problem for $\mathbf{Z}$ would be solvable. Q.E.D.

Proof of Proposition 1. If $S$ satisfies the conditions of the proposition, then

$$
z \in \mathbf{Z} \leftrightarrow \exists Z \in R[T]:(\operatorname{Imt}(Z) \wedge Z \sim z \wedge \in S) .
$$

Moreover, if $R$ contains $\mathbf{Q}$, then we define $S$ by:

$$
x \in S \leftrightarrow x \in R[T] \wedge(x=0 \vee \exists y \in R[T]: x y=1) \text {. Q.E.D. }
$$

3. Fields of rational functions. Let $F$ be a field. A projective curve $E$, given by the affine equation $c y^{2}=x^{3}+a x+b$, is called an elliptic curve defined over $F$ if it is nonsingular and if $a, b$ and $c$ are in $F$. One defines (see, e.g., Cassels [3, §7], Fulton [11, Chapter 5, §6] or Lang [12, Chapter 1, §\$3, 4]) a commutative group law " + " on the set $E(F)$ of points on the elliptic curve $E$ which are rational over $F$. The neutral element of this group is the unique point $\underline{0}$ at infinity on $E$. We shall denote by $(v, w)$ the point with affine coordinates $x=v, y=w$.

Every elliptic curve $E$ defined over $\mathbf{Q}$ whose $j$ invariant $\left(j=2^{8} 3^{3} a^{3} /\left(4 a^{3}+\right.\right.$ $\left.27 b^{2}\right)$ ) is not integral has no complex multiplication, i.e. the only $\mathbf{C}$-rational maps from $E$ into itself which fix $\underline{0}$ are the maps $P \mapsto m \cdot P=P+P$ $+\cdots+P$ ( $m$ times), $m \in \mathbf{Z}$. (See, e.g., Lang [12, Chapter $1, \S 5$ and Chapter 5, §2, Theorem 4].)

From now on we fix an elliptic curve $E_{0}$ defined over $\mathbf{Q}$, without complex multiplication and with equation

$$
y^{2}=x^{3}+a x+b
$$

To $E_{0}$ we associate the elliptic curve

$$
\left(T^{3}+a T+b\right) Y^{2}=X^{3}+a X+b,
$$

defined over $Q(T)$, which we denote from now on by $E$. Obviously the point $P_{1}$ with coordinates $(T, 1)$ lies on $E(\mathbf{Q}(T))$. 
Let $K$ be any field of characteristic zero; then we have

LEMMA 3.1. The point $P_{1}$ is of infinite order and generates the group $E(K(T))$ modulo points of order two.

Proof. We identify $T$ with the rational function $(x, y) \mapsto x$ on $E_{0}$ and we denote the rational function $(x, y) \mapsto y$ on $E_{0}$ by $U$. The function field $F$ of $E_{0}$ over $K$ is thus $F=K(T, U)$, where $U^{2}=T^{3}+a T+b$. Let $\psi_{1}$ be the $F$-rational map

$$
\psi_{1}: E \rightarrow E_{0}:(X, Y) \mapsto(X, U Y) .
$$

Notice that $\psi_{1}$ is a group homomorphism since it is rational and $\psi_{1}(\underline{0})=\underline{0}$. We denote the group of $K$-rational maps from $E_{0}$ into $E_{0}$ by $\operatorname{Rat}_{K}\left(E_{0}, E_{0}\right)$. Let $\psi_{2}$ be the map

$$
\psi_{2}: E_{0}(F) \rightarrow \operatorname{Rat}_{K}\left(E_{0}, E_{0}\right)
$$

which sends the point $(V, W)$ on $E_{0}(F)$ to the $K$-rational map

$$
\psi_{2}(V, W): E_{0} \rightarrow E_{0}:(x, y) \mapsto(V(x, y), W(x, y)) .
$$

Obviously $\psi_{2}$ is a homomorphism. Consider the group homomorphism

$$
\psi=\psi_{2} \circ \psi_{1}: E(K(T)) \rightarrow \operatorname{Rat}_{K}\left(E_{0}, E_{0}\right) .
$$

For all points $(X, Y)$ on $E(K(T))$ we have

$$
\begin{gathered}
T \circ \psi(X, Y)=X, \\
U \circ \psi(X, Y)=U Y .
\end{gathered}
$$

Hence $\psi$ is injective. Since $E_{0}$ has no complex multiplication, we have

$$
\operatorname{Rat}_{K}\left(E_{0}, E_{0}\right) \cong\left\{\alpha_{m} \mid m \in \mathbf{Z}\right\} \oplus E_{0}(K),
$$

where $\alpha_{m}$ is the map $P \mapsto m \cdot P$, and where we identify a point on $E_{0}$ with the constant map from $E_{0}$ onto this point. Notice that $\psi\left(P_{1}\right)=\alpha_{1}$, and $\psi\left(m \cdot P_{1}\right)$ $=\alpha_{m}$. Thus $P_{1}$ is of infinite order. Moreover, if $(X, Y) \in E(K(T))$ and $\psi(X, Y) \in E_{0}(K)$, then $X \in K$ by (3) and (2) yields $Y=0$. This means that $(X, Y)$ is a point of order two on $E(K(T))$. The lemma follows now from (5). Q.E.D.

We denote, for any nonzero integer $m$, the affine coordinates of $m \cdot P_{1}$ by $\left(X_{m}, Y_{m}\right)$. Notice that $X_{m}$ and $Y_{m}$ are in $\mathbf{Q}(T)$. For any $V$ and $W$ in $K(T)$ we write $V \sim W$ to denote that $V-W$ (considered as a rational function on the projective line over $K$ ) takes the value zero at infinity.

LEMMA 3.2. Using the above notation we have $X_{m} / T Y_{m} \sim m$ for all nonzero integers $m$.

Proof. Notice that $T / U$ is a local parameter on $E_{0} a_{\iota} \underline{0}$, hence 


$$
\left\{\frac{(T / U) \circ \alpha_{m}}{T / U}\right\}(\underline{0})=m \text {. }
$$

(See, e.g., Lang [12, Appendix 1, §3].) On the other hand, from (3) and (4) follows

$$
\frac{X_{m}}{T Y_{m}}=\frac{T \circ \psi\left(X_{m}, Y_{m}\right)}{\left(U \circ \psi\left(X_{m}, Y_{m}\right)\right) T / U}=\frac{T \circ \alpha_{m}}{\left(U \circ \alpha_{m}\right) T / U}=\left(\frac{T}{U} \circ \alpha_{m}\right) / \frac{T}{U}
$$

Q.E.D.

Let us define the 1-ary relation $\operatorname{Imt}(Z)$ in $K(T)$ by

$$
\operatorname{Imt}(Z) \leftrightarrow Z \in K(T)
$$

$$
\wedge\{Z=0 \vee \exists X, Y \in K(T):((X, Y) \in 2 \cdot E(K(T)) \wedge 2 T Y Z=X)\}
$$

LEMMA 3.3. (i) The relation $\operatorname{Imt}(Z)$ is diophantine over $K(T)$ with coefficients in $\mathbf{Z}[T]$.

(ii) If $Z$ satisfies $\operatorname{Imt}(Z)$, then there exists an integer $m$ such that $Z \sim m$.

(iii) For every integer $m$, there exists an element $Z$ in $Q(T)$ satisfying $\operatorname{Imt}(Z)$ and $Z \sim m$.

Proof. This follows at once from Lemmas 3.1 and 3.2.

We consider the relation $\operatorname{Com}(y)$ defined by

$$
\operatorname{Com}(y) \leftrightarrow y \in K(T) \wedge \exists x \in K(T): y^{2}=x^{3}-4
$$

The following lemma was used by R. M. Robinson [22, §4] too:

LEMMA 3.4. (i) The relation $\operatorname{Com}(y)$ is diophantine over $K(T)$ with coefficients in $\mathbf{Z}$.

(ii) If $y$ satisfies $\operatorname{Com}(y)$, then $y$ lies in $K$.

(iii) For every rational number $z$, there exists a rational number $y$ satisfying $\operatorname{Com}(y)$ and $y>z$.

(iv) If $K$ contains the real closure of $\mathbf{Q}$, then every integer $y$ satisfies $\operatorname{Com}(y)$.

Proof. (i) and (iv) are obvious.

(ii) Since $y^{2}=x^{3}-4$ is a curve of genus 1 , it admits no rational parametrization.

(iii) It is known (see, e.g., R. M. Robinson [22, \$4]) that the group of rational points on the elliptic curve $y^{2}=x^{3}-4$ is infinite. So the rational points are everywhere dense on the curve in the real plane. Indeed since the curve is connected in the real plane, its group of real points is a topological group isomorphic to the circle group. But every infinite subgroup of the circle group is everywhere dense. Q.E.D.

We define the 1 -ary relation $Z \dot{\sim} 0$ in $K(T)$ by 


$$
\begin{aligned}
& Z \dot{\sim} 0 \leftrightarrow Z \in K(T) \wedge \exists X_{1}, X_{2}, X_{3}, X_{4}, X_{5}, y \in K(T): \\
& (\operatorname{Com}(y) \wedge \\
& \left.\quad(y-T) Z^{2}+1=X_{1}^{2}+X_{2}^{2}+X_{3}^{2}+X_{4}^{2}+X_{5}^{2}\right) .
\end{aligned}
$$

LEMMA 3.5. (i) The relation $Z \dot{\sim} 0$ is diophantine over $K(T)$ with coefficients in $\mathbf{Z}[T]$.

(ii) If the field $K$ is formally real and if $Z \dot{\sim} 0$, then $Z \sim 0$

(iii) If $Z \in \mathbf{Q}(T)$ and $Z \sim 0$, then $Z \dot{\sim} 0$.

Proof. (i) is obvious.

(ii) Suppose there exist $X_{1}, \ldots, X_{5}, y$ in $K(T)$ satisfying (6) and (7). Suppose we have not $Z \sim 0$, then $\operatorname{deg} Z \geqslant 0$ (where $\operatorname{deg} Z$ denotes the degree of the rational function $Z$ ). From (6) and Lemma 3.4(ii) follows $y \in K$. Hence $\operatorname{deg}\left((y-T) Z^{2}+1\right)$ is positive and odd. But deg $\left(X_{1}^{2}+X_{2}^{2}\right.$ $\left.+\cdots+X_{5}^{2}\right)$ is even. Indeed there is no cancellation of the coefficients of largest degree, since a sum of squares in a formally real field vanishes only if each term is zero. So we are in contradiction with (7), hence $Z \sim 0$.

(iii) Let $Z \in \mathbf{Q}(T)$ and $Z \sim 0$, then $T Z^{2} \sim 0$ and there is a natural number $z$ such that

$$
\left|\left(T Z^{2}\right)(r)\right| \leqslant \frac{1}{2} \quad \text { when } r \in \mathbf{R} \text { and }|r|>z .
$$

By Lemma 3.4(iii) there exists a rational number $y$ satisfying $\operatorname{Com}(y)$ and $y>z \geqslant 0$. Thus

$$
\left((y-T) Z^{2}+1\right)(r) \geqslant 0 \text { for all } r \text { in } \mathbf{R} .
$$

But a theorem of $Y$. Pourchet [16] states that every positive definite rational function over $\mathbf{Q}$ can be written as a sum of five squares in $Q(T)$. Hence there exist $X_{1}, \ldots, X_{5}$ in $K(T)$ satisfying (7), whence $Z \dot{\sim} 0$. Q.E.D.

PROOF OF THEOREM B. There exists an algorithm to find for any polynomial $P\left(z_{1}, \ldots, z_{n}\right)$ over $\mathbf{Z}$, a polynomial $P^{*}\left(Z_{1}, \ldots, Z_{m}\right)$ over $\mathbf{Z}[T]$ such that

$$
\begin{aligned}
\exists z_{1}, \ldots, z_{n} \in \mathbf{Z}: & P\left(z_{1}, \ldots, z_{n}\right)=0 \\
& \leftrightarrow \exists Z_{1}, \ldots, Z_{m} \in K(T): P^{*}\left(Z_{1}, \ldots, Z_{m}\right)=0 .
\end{aligned}
$$

Indeed, by Lemmas 3.3 and 3.5 we have

$$
\begin{aligned}
\exists z_{1}, \ldots, z_{n} \in \mathbf{Z}: P\left(z_{1}, \ldots, z_{n}\right) & =0 \leftrightarrow \exists Z_{1}, \ldots, Z_{n} \in K(T): \\
\left(\operatorname{Imt}\left(Z_{1}\right)\right. & \left.\wedge \cdots \wedge \operatorname{Imt}\left(Z_{n}\right) \wedge P\left(Z_{1}, \ldots, Z_{n}\right) \dot{\sim} 0\right) .
\end{aligned}
$$

Proceed now as in the proof of Theorem A. Q.E.D.

Proof of Proposition 2. If $S$ satisfies the conditions of the proposition, then

$$
z \in \mathbf{Z} \leftrightarrow \exists Z \in K(T):(\operatorname{Imt}(Z) \wedge Z-z \dot{\sim} 0 \wedge z \in S)
$$


Moreover, the last assertion of the proposition follows from Lemma 3.4(iv). Q.E.D.

\section{REFERENCES}

1. J. Ax, On the undecidability of power series fields, Proc. Amer. Math. Soc. 16 (1965), 846.

2. J. Becker and L. Lipshitz, Remarks on the elementary theories of formal and convergent power series, Fund. Math. (to appear).

3. J. W. S. Cassels, Diophantine equations with special reference to elliptic curves, J. London Math. Soc. 41 (1966), 193-291.

4. M. Davis, Hilbert's tenth problem is unsolvable, Amer. Math. Monthly 80 (1973), 233-269.

5. M. Davis and H. Putnam, Diophantine sets over polynomial rings, Illinois J. Math. 7 (1963), 251-256.

6. M. Davis, Yu. Matijasevic and J. Robinson, Diophantine equations: Positive aspects of a negative solution, Proc. Sympos. Pure Math., vol. 28, Amer. Math. Soc., Providence, R.I., 1976, pp. 323-378.

7. J. Denef, Hilbert's tenth problem for quadratic rings, Proc. Amer. Math. Soc. 48 (1975), 214-220.

8. _ Diophantine sets over Z[T], Proc. Amer. Math. Soc. 69 (1978), 148-150.

9. Yu. Eršov, Undecidability of certain fields, Dokl. Akad. Nauk SSSR 161 (1965), 349-352.

10. __ New examples of undecidable theories, Algebra i. Logika 5 (1966), 37-47.

11. W. Fulton, Algebraic curves, Benjamin, New York, 1969.

12. S. Lang, Elliptic functions, Addison-Wesley, London, 1973.

13. L. Lipshitz, Undecidable problems for addition and divisibility in algebraic number rings. II, Proc. Amer. Math. Soc. 64 (1977), 122-128.

14. A. I. Malcev, On the undecidability of the elementary theories of certain fields, Sibirsk Mat. Z. 1 (1960), 71-77; ibid 2 (1961), 639; English transl., Amer. Math. Soc. Transl. (2) 48 (1965).

15. Yu. Penzin, Undecidability of fields of rational functions over fields of characteristic 2, Algebra i. Logika 12 (1973), 205-210; 244.

16. Y. Pourchet, Sur la représentation en somme de carrés des polynômes à une indéterminé sur un corps de nombres algébriques, Acta Arith. 19 (1971), 89-104.

17. J. Robinson, Definability and decision problems in arithmetic, J. Symbolic Logic 14 (1949), 98-114.

18. _ The undecidability of algebraic rings and fields,Proc. Amer. Math. Soc. 10 (1959), 950-957.

19. On the decision problem for algebraic rings, Studies in Math. Anal. and Related Topics, Stanford, 1962, pp. 297-304.

20. Amsterdam, 1965, pp. 299-311.

21. R. M. Robinson, Undecidable rings, Trans. Amer. Math. Soc. 70 (1951), 137-159.

22. __ The undecidability of pure transcendental extensions of real fields, $\mathbf{Z}$. Math. Logik Grundlagen Math. 10 (1964), 275-282.

23. A. Tarski, The elementary undecidability of pure transcendental extensions of real closed fields, Notices Amer. Math. Soc. 10 (1963), A-355.

University of Leuven, Department of Mathematics, Celestunenlaan 200B, 3030 HeVERLEe, BeLGiUM

Current address: Department of Mathematics, Princeton University, Fine Hall, Box 37, Princeton, New Jersey 08540 